\title{
A descriptive study on risky sexual behaviors of men who have sex with men and the impact of counseling services on behavior change, in a sexually transmitted diseases clinic in Sri Lanka
}

\author{
Ranatunga $\mathbf{J}^{1}$, Karawita $\mathrm{DA}^{2}$, Batagalla PSK $^{3}$, Perera WPP4 , Senevirathne $\mathrm{MR}^{4}$, Thilakaratne \\ PTMIS ${ }^{4}$
}

\begin{abstract}
Introduction: Sri Lanka is considered a low prevalence country for HIV since the first case was diagnosed in 1986. In the recent years, the country is experiencing an increasing incidence of HIV among young men who have sex with men (MSM). A record based baseline study was first conducted to collect the demographic and behavioral data among the group. Based on these findings, a self- administered questionnaire was then used at the first visit and 6 months later to assess the effectiveness of routine counseling provided in the sexually transmitted diseases clinic (STD clinic) on changing their behavior and attitudes.
\end{abstract}

\section{Method:}

A study was conducted first to establish baseline data among the group and based on the findings; it was studied further by a descriptive study to assess the effectiveness of routine counseling done in STD clinic on changing their behavior and attitudes. The baseline survey was a record based study while the proper study was conducted among 82 MSM's attending the clinic, using a self-administered questionnaire at the first visit and 6 months after to assess the behavior change.

\section{Results:}

Seventy nine percent were aware about the condom as a preventive device. Only $54.8 \%$ used a condom at their last sex. Sixty three percent had a reluctance to attend the clinic for the first time. Ninety four percent said they were well accepted by both the minor and major staff. Ninety three percent of them thought screening for STIs is important after the first counseling session and $89 \%$ of them thought bringing the partners to the clinic for screening is important. Seventy nine percent said that they realized attending the clinic again is very important and they will do that. Ninety six percent of them accepted that they gained knowledge on HIV and STIs. After 6 months, $89 \%$ accepted that they changed their attitudes regarding attending STI services. Ninety percent of them wanted to change their risky behavior after routine counseling at STI services but only $74.4 \%$ of them reported a behavior change of some means.

\section{Conclusions:}

Although counseling is done aimed at reducing risky behavior and attitudes of MSMs on seeking regular health care including screening, the change is not up to the expected level of at least $80 \%$. The attendance to clinics after the first visit is very low. Contact screening is also at a very low level.

\section{Key words:}

Sexual risk, behaviour change, HIV, MSM, Sri Lanka, counseling, condom use

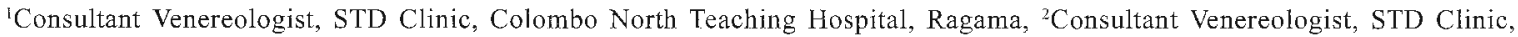
Anuradbapura, ${ }^{3}$ Registrar in Venereology, National STD/AIDS Control Programme, ${ }^{4}$ Medical officer, STD Clinic, Colombo North Teaching Hospital, Ragama

Corresponding author: Ranatunga J, Email: jayadarie@yahoo.com
} 


\section{Introduction}

Sri Lanka remains as a low HIV prevalent country with an estimated sero-prevalence of less than $0.1 \%$ in the general population ${ }^{1}$. The sero-prevalence among key populations (female sex workers (FSW), MSM, injecting drug users (IDU) and beach boys) is also $<0.1 \%{ }^{1}$. Among the most at- risk groups, MSM are an important group to address because of their pattern of risky sexual behavior. In the past few years, there has been increased concern about new, newly identified and resurging epidemics of HIV infection in MSM on a global level ${ }^{2,3}$. Over the past years, it is observed that the prevalence of sexually transmitted infections especially syphilis is increasing among MSMs all over the world. Research among MSM in low and middle income countries has been limited due to the result of criminalization and social stigmatization of these behaviors.

Change of risky sexual behavior is the key to reducing new infections among these populations. Behavior change communication is the mainstay of the behavior change process. At STD clinics in Sri Lanka, we offer standard counseling for HIV testing and a formal one-on-one interview with a trained health care worker to support people with risky sexual behaviors to change their behaviors.

The main objective of this study was to ascertain the effectiveness of counseling done at the STD clinic, Ragama on changing attitudes and sexual risk behaviors among MSM. Furthermore, the study specifically looked at the level of HIV risk behaviours, attitudes towards government STD clinic services and effectiveness of behavior change communication.

We observed a significant increase of MSMsattending the STD clinic at Teaching Hospital Ragama with STIs and HIV during the recent years. The percentages of HIV diagnosis among MSMs who attended the Ragama Clinic was $1.75 \%(1 / 57)$, $3.22 \%(1 / 31), 5.26 \%(1 / 19)$ and $3.09 \%$ ( $3 / 97)$ in years $2009,2010,2011$ and 2012 respectively. The percentages of STI s reported among MSMs were also significant amounting to $61.4 \%, 58 \%, 68.4 \%$ and $44.3 \%$ in 2009,2010,2011 and 2012 respectively. As this was a new observation in comparison to the previous years, which may be indicative of a drastic change in the country's epidemic, we conducted a baseline assessment of their sexual behavior. The findings were very significant with $42 \%$ having casual partners and $21 \%$ having non-regular partners; their condom use at last exposure was only $13 \%$.

\section{Methodology}

In 2012, a baseline assessment on the HIV risk behaviors among MSMs who attended the STD Clinic, Ragama was conducted ${ }^{4}$. Based on the alarming findings, we wanted to assess the impact of counseling services provided by the STD clinic Ragama. So a descriptive cross sectional study was carried out on the MSMs who attend the STD Clinic

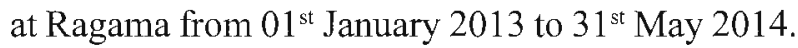
All the consecutive MSMs registered in the clinic during the specified period were recruited to the study.A questionnaire was introduced at the first visit to get the baseline data according to the objectives of the study. A separate questionnaire was given to the same subjects at 6 months to assess behavior change overtime and the change in the attitudes and perceived barriers to reach the services provided by the government STD clinics.

Even though 194 MSMs were registered in the clinic during the study period, only 82 subjects attended the clinic for a follow up visit at six months. So, the results were analyzed only using the sample who responded after 6 months, by using the Statistical Package for the Social Sciences (SPSS) version 16.

\section{Results}

\section{Background characteristics}

Only $82(42.2 \%)$ attended the clinic after 6 months for a review at the clinic. Out of the number who attended after 6 months, majority $(53.7 \%)$ was between 21-30 years of age, $8.6 \%$ belonged to the age group of $15-20$ years while only a minority $(2.4 \%)$ was above the age of 50 . Sixty two $(75.6 \%)$ of them 
were single while $16(19.5 \%)$ were married. Majority (42.7\%) was educated up to $\mathrm{O} / \mathrm{L}$ and $14.6 \%$ had only primary education. A small percentage $(6.1 \%)$ had a degree or a diploma. A major percentage $(56.1 \%)$ of them was employed with wages while $13.4 \%$ were unemployed. Nineteen percent of them were self-employed.

Half $(50 \%)$ of the sample reported observing people was their method of finding partners while $35.4 \%$ reported friends as the way of finding partners. Out of the MSM studied, the majority (35.4\%) were brought to the clinic by the escorts carried out by the peer led programme conducted in the district. Only a very small percentage $(3.7 \%)$ attended the clinic voluntarily. While $19.5 \%$ of them attended the clinic with symptoms, $13.4 \%$ came to the clinic by a referral. It was interesting to note that more than half $(53.7 \%)$ of them did have an STI on attendance.

\section{Level of risk behaviour}

A majority (79.3\%) of them did know about the condom before coming to the clinic while $20.7 \%$ of them were unaware. Only $51.2 \%$ of them had knowledge on lubricants. Out of the total sample of 82 , only $45(54.8 \%)$ used a condom at their last sexual exposure. The majority $(57.3 \%)$ of them were nonalcohol users. A significant number, 14 (17.1\%) reported habitual use of alcohol. while $93.9 \%$ of them were non - recreational drug users.

\section{Attitudes towards government STD services}

More than half of them $(63.4 \%)$ had a reluctance to attend the clinic for the first time while $24(29.3 \%)$ reported that they did not have any reluctance. Majority (93.7\%) of them said they were well accepted by both the minor and major staff including the doctors who worked as counselors and they did not have any concerns regarding the confidentiality while only $1.2 \%$ of them felt insecure with the STD clinic staff. Majority (92.7\%) of them thought screening for STI's is important after the counseling session and $89 \%$ of them thought bringing the partners to the clinic for screening is important. Majority (79.3\%) of them said that they realized attending the clinic again is very important and they will do that. Only $4.9 \%$ thought it is not important. Majority (96.3\%) of them accepted that they gained knowledge on HIV and STIs after the clinic visit.

\section{Effectiveness of behavior change communication}

When we analyzed the sample after 6 months, the majority $(89 \%)$ accepted that they changed their attitudes regarding attending STI services while only $11 \%$ said that their attitudes were not changed. But a great majority $(90.2 \%)$ of them wanted to change their risky behavior after routine counseling at STI services. $9.8 \%$ of them did not want to change their behavior. Although $90.2 \%$ were willing to change their behavior, only $74.4 \%$ of them accepted that a behavior change has occurred.

\section{Discussion}

Behaviour change through counseling is the mainstay to reduce risky sexual behaviours and thereby reduce the transmission of HIV infection. The services provided in STD clinics in Sri Lanka provide standard HIV test counseling as well as counseling on safer sexual practices for the clients. This study was conducted on the findings of baseline study done in 2012 and was focused to assess the level of risky sexual behaviours, attitudes among MSM towards government STD clinics and the effectiveness of behaviour change communication done in the STD clinic settings.

\section{Socio- demographic factors}

The survey showed that their literacy level is lower than the baseline survey showing the efficacy of the peer led programme to reach lower social strata as well. It is very important to reach this population as they are the most vulnerable and will not attend the services. The fact that a major percentage $(56.1 \%)$ of them were employed with wages should be taken in to consideration for making the clinic opening hours friendly to them.. None from this group reported commercial sex as their method of livelihood while $4.1 \%$ from the previous group reported commercial sex. 


\section{Level of risk behaviours}

The rate of anal sex was significantly high and the condom use at last sex was $54.8 \%$. This study showed an improved rate for condom use at last sex when compared with the baseline survey which was only $10 \%$. UNGASS country progress reports in 2008 also showed similar results with $54 \%$ condom use at last sexual exposure ${ }^{5}$. This higher figure may be due to the peer led programme. None of them reported condom use with oral sex. Only $51.2 \%$ of them had some knowledge on lubricants. In contrast, a study done among Latina MSM in New York showed very high rate of knowledge and usage on lubricants ${ }^{6}$.

The presence of STIs in more than half of the sample was a significant factor increasing the acquisition of HIV. The use of alcohol and recreational drugs by them though the percentage is small, is contributory to increasing the risk of acquisition of HIV. A study done in a London sexual health clinic showed that although counselling reduces the immediate risk taking behaviour, it does not affect the rate of acquisition of new infections among high risk groups?

The analysis of reason for attendance showed us that both voluntary attendance and referrals were low. This factor should be taken in to consideration to improve knowledge of care providers on symptoms of STIs and link between high risk groups and STIs. A major percentage $(93.9 \%)$ of them was nonrecreational drug users. A significant number 14 $(17.1 \%)$ reported habitual alcohol use. A similar study done in china revealed similar alcohol usage rate and higher illegal drug use ${ }^{8}$.

HIV transmission and acquisition is high in the presence of an STI. It was interesting to note that more than half $(53.7 \%)$ of them did have an STI on attendance. In the above study among Chinese MSM, only $20 \%$ were diagnosed to be having an STI ${ }^{8}$.

\section{Attitude towards government STD services}

Perceived stigma in clinical settings is the main factor discouraging high risk groups attending health care facilities.
In this study, more than half of them $(63.4 \%)$ were reluctant to attend the clinic when interviewed in the first visit. But the majority (93.7\%) of them said they were well accepted by both the minor and major staff. The well-structured training programmes including counselling for STD clinic staff should be done regularly. A similar study conducted in Nigeria showed poor attitude of staff towards them ${ }^{9}$.

Compared to studies carried out in Belize ${ }^{10}$ and Los Angeles ${ }^{11}$, the discrimination by the health care staff is minimal at the study setting. While the majority $(92.7 \%)$ of them thought screening for STI's is important after the counseling session and the fact that most $(89 \%)$ of them thought that bringing the partners to the clinic for screening is important should be studied in detail ideally by a qualitative survey as actual partner screening is very low at the Ragama Clinic as well as in all STI clinics in Sri Lanka.It was very satisfying and encouraging to notice that most (96.3\%) of them said that they gained knowledge on HIV and STIs after the clinic visit.

\section{Effectiveness of behaviour change communication}

A main objective of this study was to find out the change in risky behaviour after a period of time from the first presentation to the clinic. In this study only $42.2 \%$ attended the clinic for the second time which is lower than the expected.

A study done at an anonymous testing site in San Francisco, California on counselling interventions has given promising results in decreasing individual risk behaviour and thus limiting community-level HIV transmission ${ }^{13}$.A multi-center assessment on the effect of counselling on negative consequences can reduce HIV transmission behaviours significantly ${ }^{14}$.

Although the majority (74.4\%) verbally accepted a behaviour change, a low $(54.8 \%)$ condom use at last sex was noted. The knowledge on lubricant use $(51.2 \%)$ to protect the condom shows the efficiency of the peer led model to educate high risk groups. When we analysed the sample after 6 months, the majority (89\%) accepted that they changed their attitudes regarding attending STI services.Only 9.8\% of them did not want to change their behaviour. 
The investigators of this study wanted to assess the behaviour change of a major risk group in the country with regular counselling services done in the government STI services and to make necessary changes which will have an impact in changing the country epidemic. The study was done in only one clinic as the investigators were based at the study site. This result could be generalized as the medical officers and other staff working at STI clinics in Sri Lanka is given a standard training from the national programme on behaviour change counseling.

\section{Conclusion}

Although counseling is done aimed at reducing risky behavior and attitudes of MSMs on seeking regular health care including screening, the change is not up to the expected level of at least $80 \%$. The attendance to clinics after the first visit is very low. Contact screening is also at a very low level.

\section{Recommendations}

Training of health care workers on counseling for change in sexual behavior of MSM should be a continuous process and the modules should be updated based on evidence. Measures should be taken to improve contact and default tracing. Health care providers should be given knowledge on STI, $\mathrm{HIV}$, and high risk groups in order to improve referrals. Qualitative research should be done in order to assess the factors for continuing risky behavior despite the knowledge.

\section{Acknowledgements}

We acknowledge all the patients who participated in this study, the staff of the STD Clinic, Ragama and Sri Lanka College of Venereologists for giving hands to make this study a success.

\section{Conflict of interest}

Authors declare no conflict of interest

\section{Funding}

No specific funding assistance

\section{References}

1. HIV Sentinel Sero-surveillanceSurvey in Sri Lanka.(May 2009). Colombo: National STD/ AIDS Control Programme, Ministry of Health

2. BaralS, Sifakis F, Cleghorn F, et al.Elevated riskfor HIV infection among men who have sex with men in low- and middle-income countries 2000-2006: a systematic review. PLoS Med 2007;4:e339.

3. vanGriensven F, van Wijngaardenc JW, Baral S, et al. The global epidemic of HIV infection among men who have sex with men. CurrOpin HIV AIDS 2009;4:300-7.

4. Ranathunga RGJD, Karawita DA, Batagalla PSK, et al. HIV risk behaviors among men who have sex with men (MSM) who attend STD Clinic Ragama. The Sri Lanka Journal of Venereology, 2013;4:13-18

5. Philippe Adam, John de Wit, Igor Toskin, et al. Estimating levels of HIV testing, HIV prevention coverage, HIV knowledge, and condom use among men who have sex with men (MSM) in lowIncome and middle-income countries. Journal of Acquired Immune Deficiency Syndromes, December 2009; 52:S143-S151. doi: 10.1097/QAI.0b013e3181baf111

6.A Carballo-Diéguez, Z Stein, H Sáez, et al Frequent use of lubricants for anal sex among men who have sex with men: the HIV prevention potential of a microbicidal gel. Am J Public Health. 2000 July; 90(7): 1117-1121

7. John Imrie, Judith M Stephenson, Frances M Cowan, ShamilWanigaratne et al. A cognitive behavioural intervention to reduce sexually transmitted infections among gay men: randomized trial. BMJ 2001;322:1451. doi: http://dx.doi.org/ $.1136 / \mathrm{bmj} .322 .7300 .1451$

8. Yuji Feng, Zunyou Wu, Roger Detels, Guangming Qin, et al HIV prevalence among MSM in Chengdu, China and associated risk factors for HIV infection. J Acquir Immune DeficSyndr. Feb 2010;53(Suppl 1): S74-S80.doi: 10.1097/ QAI.0b013e3181c7dd16 
9.BolanleAdebajo S, BamgbalaAO, and Oyediran MA, Attitudes of health care providers to persons living with HIV/AIDS in Lagos State, Nigeria. 2003; African Journal of Reproductive Health.

10.Aisha Andrewin and Li-Yin Chien. AIDS Patient Care and STDs. November 2008, 22(11): 897906. doi:10.1089/apc.2007.0219

11.Kinsler JJ, Wong MD, Sayles JN, et al. AIDS Patient Care and STDs. August 2007, 21(8): 584592. doi:10.1089/apc.2006.0202.

12.Picciano J, Roffman R, Kalichman S, et al. A telephone based brief intervention using motivational enhancement to facilitate HIV risk reduction among MSM: A Pilot Study. AIDS and behavior. 2001;5(3):251-62.

13.Dilley JW, Woods WJ, Sabatino J et al. Journal of Acquired Immune Deficiency Syndromes. (1999); [2002, 30(2):177-186]

14.Richardson JL, Joel M, Allen M et al. AIDS.( may 2004); [18( 8):1179-1186] 